\title{
Aortic stenosis - pathogenesis, prediction of progression, and percutaneous intervention
}

\author{
D Natarajan ${ }^{1}$, B Prendergast ${ }^{2}$
}

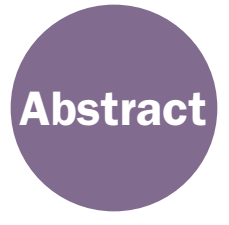

Aortic stenosis is common and an important cause of morbidity and mortality. Prevalence will increase significantly in forthcoming decades as a function of the ageing population; treatment by means of surgery or percutaneous intervention is expensive. Epidemiological, mechanistic and interventional studies are therefore vital to determine optimal and innovative treatments and their funding.

Recent studies suggest that aortic stenosis is not a passive degenerative disease, but an active process involving several pathways, including lipid infiltration, chronic inflammation, fibrosis formation, osteoblast activation, and active valve mineralisation. Despite similarities with atherosclerosis, randomised statin trials proved negative in aortic stenosis, underlining the need to explore alternative pathophysiological pathways.

Left ventricular hypertrophy in response to pressure overload in aortic stenosis is initially adaptive but ultimately decompensates, leading to progressive left ventricular impairment, symptoms and adverse cardiovascular events. This transition is driven primarily by myocyte death and myocardial fibrosis. Cardiac magnetic resonance imaging can visualise and quantify myocardial fibrosis and may provide additional and independent prognostic information in aortic stenosis. Moreover, new markers of fibrosis utilising novel imaging techniques are rapidly emerging.

Transcatheter aortic valve implantation is a disruptive technology that has transformed the management of aortic stenosis, and encouraged a wider multidisciplinary approach to the management of valvular heart disease. While originally applied in older, high-risk patients, recent trends for its use in intermediate risk patients have been supported by the findings of key clinical trials in 2016.

Keywords: aortic stenosis, aortic valve replacement, biomarkers, pathogenesis, transcatheter aortic valve implantation, treatment outcome

Declaration of Interests: No conflict of interests declared

\section{Correspondence to:}

B Prendergast

Department of Cardiology

St Thomas' Hospital

London

UK

\section{Email:}

bernard.prendergast@gstt. nhs.uk

Based on a lecture given at the Cardiology symposium, Royal College of Physicians of Edinburgh, November 2016

\section{Introduction}

Aortic stenosis (AS), first described in 1663 by Lazare Riviere who noted left ventricular (LV) enlargement and carunclelike masses obstructing the LV outflow tract, ${ }^{1}$ is currently the most prevalent native valve pathology in the Western world. ${ }^{2}$ The disease is characterised by a bimodal clinical course with an initial long quiescent phase followed by rapid clinical deterioration and high mortality once symptoms such as angina, syncope or heart failure develop. ${ }^{3}$ However, more recent studies demonstrate that outcome is worse than previously assumed in patients with mild to moderate AS, and that rapid progression to severe stenosis is not uncommon. ${ }^{4}$ Moreover, even patients with so called 'aortic sclerosis' (initially considered a benign condition) experience significant medical and surgical events over 20 year follow- up. ${ }^{5}$ Despite high prevalence and major impact on morbidity and mortality, there is no effective evidence-based medical therapy.

\section{Pathogenesis}

AS is characterised by progressive aortic valve narrowing and secondary LV hypertrophy. ${ }^{6}$ The earliest pathophysiological stages are characterised by endothelial damage, lipid deposition, and inflammation, and share many similarities with atherosclerosis. Subsequent progressive calcification due to increased osteoblast activity within the valve results in leaflet stiffening, reduced separation and valve narrowing. Despite promising pre-clinical data, several randomised controlled trials, including the Scottish Aortic Stenosis and Lipid Lowering Trial Impact on REgression (SALTIRE) study, 
have demonstrated that lipid lowering has no impact on the progression of calcific AS. ${ }^{7}$ As a consequence, studies investigating the effects of pharmacological manipulation of calcium pathways in patients with mild and moderate AS are now underway.

AS increases pressure afterload, resulting in LV wall stress and hypertrophy. The combination of these mechanical forces with insufficient myocardial oxygen supply (as a result of increased myocardial mass, afterload and an insufficient coronary capillary network) leads to increased apoptosis in the hypertrophied ventricle, ${ }^{6,8-11}$ fibrosis and scarring with eventual progressive LV dysfunction ${ }^{12}$ and rapid clinical deterioration.

\section{Predictors of progression and the timing of intervention}

In patients with asymptomatic severe AS, guidelines currently recommend close clinical monitoring for the development of symptoms prior to consideration of aortic valve intervention. However, the long-term outcome of conservative management is often poor and these patients may be better served by an earlier interventional strategy. ${ }^{13}$ The current challenge is to locate the 'sweet spot', allowing identification of patients on the verge of developing symptoms (or asymptomatic LV decompensation) who will then benefit from prompt intervention. Rosenhak et al. ${ }^{14}$ have already demonstrated the value of echocardiography in predicting the outcome of asymptomatic patients with severe AS, whereby event-free survival rates were worse for patients with a higher peak aortic jet velocity. More recently, Chin et al. have reported an association of plasma cardiac troponin I (cTnl) concentration with advanced hypertrophy and mid-wall myocardial fibrosis, ${ }^{15,16}$ as well as the important clinical endpoints of cardiovascular death or need for aortic valve replacement. As such, it has been suggested that cTnl (and other putative biomarkers, such as B-type natriuretic peptide) may play a role in the early recognition of the transition from hypertrophy to heart failure. ${ }^{17,18}$ The forthcoming EVOLVED-AS study will investigate whether a strategy of screening using a combination of chemical, ECG and imaging biomarkers is able to identify at-risk patients with asymptomatic moderate-severe AS who will benefit from early intervention.

\section{Transcatheter aortic valve intervention}

The number of elderly is growing. The OxVALVE population cohort study ${ }^{19}$ demonstrated that previously undetected valvular heart disease affects half of the elderly population and predicts a significant rise in the clinical impact of this emerging epidemic, with a projected two-fold increase in prevalence by 2046 . Although surgical aortic valve replacement (SAVR) is increasingly performed in the elderly population with severe $A S,{ }^{20,21}$ the presence of multiple comorbidities often precludes surgery resulting in systematic undertreatment of this at-risk population. ${ }^{20,22-25}$ An alternative to SAVR was therefore warranted.
In 2002, Cribier et al. ${ }^{26}$ reported the first human case of percutaneous prosthetic aortic valve implantation via the antegrade transseptal approach. The original valve was composed of three bovine pericardial leaflets mounted within a tubular, stainless steel balloon-expandable stent and the implantation was performed successfully in a 57-year-old male with multiple comorbidities and prohibitive surgical risk. In the wake of this pioneering intervention, a huge volume of research and technical innovation have contributed to the evolution of transcatheter aortic valve intervention (TAVI).

The PARTNER 1 Cohort B randomised controlled trial ${ }^{27}$ established that TAVI reduced mortality in comparison with medical therapy, while the companion PARTNER 1 Cohort A study ${ }^{28}$ confirmed equivalent outcomes of TAVI and SAVR in high-risk groups at long-term follow-up. Moreover, the CoreValve High-Risk trial ${ }^{29}$ demonstrated outcomes with transfemoral TAVI that surpassed the results of SAVR; this trend has been observed in other more recent studies. Not only have these trials demonstrated the non-inferiority of TAVI to SAVR in high-risk patients, they have indicated superior outcomes with transfemoral TAVI. ${ }^{30}$

The PARTNER 2 Cohort A randomised controlled trial ${ }^{31}$ compared TAVI and SAVR in intermediate-risk patients with symptomatic severe AS. The primary endpoint of all-cause mortality or disabling stroke at two years was similar in the TAVI and SAVR study groups (19.3\% vs $21.1 \%, p=0.25$ ). Importantly, a predefined sub-analysis demonstrated a clear advantage of transfemoral TAVI over SAVR (primary endpoint $16.8 \%$ vs $20.4 \%, p=0.05$ ) with favourable implications for contemporary practice where $90 \%$ of TAVI is performed via the transfemoral approach. In the parallel PARTNER S3i study, ${ }^{32}$ using the third-generation SAPIEN 3 device, rates of mortality, stroke and at least moderate paravalvular regurgitation were remarkably low at one year $(7.4 \%, 4.6 \%$ and $1.5 \%$, respectively) and superior to propensity-matched controls undergoing SAVR in the PARTNER 2 Cohort A trial.

The NOTION trial ${ }^{33}$ compared TAVI and SAVR in low-risk patients and demonstrated a trend towards reduced mortality in the TAVI cohort. Confirming the role of TAVI in younger and lower-risk patients will be addressed more comprehensively by upcoming trials such as PARTNER 3.

\section{Future direction}

TAVI has experienced phenomenal growth. Between 2007 and 2015 , almost 10,000 procedures were performed in the UK. In Germany there has been a 20-fold increase in TAVI since 2008 , from 637 to 13,264 procedures in 2014, surpassing the annual numbers of isolated SAVR since 2013 . $^{34}$ TAVI is now accessible in more than 65 countries and more than 250,000 implantations have been performed.

The increasing popularity of TAVI may be attributed in part to the progressive improvement in TAVI outcomes. Potential reasons ${ }^{30}$ include i) refinements in valve design leading to reduction in the rate of post-implantation paravalvular 
regurgitation (which had been associated with inferior outcome), ii) reduced permanent pacemaker requirements as a result of more accurate valve implantation, iii) lower-profile delivery systems resulting in reduced vascular complications and use of a fully percutaneous transfemoral approach in the majority of patients, iv) adoption of conscious sedation protocols in most centres, ${ }^{35}$ thereby avoiding the potential complications of general anaesthesia and facilitating earlier hospital discharge, and v) increasing operator experience.

\section{Conclusion}

AS is common and causes significant morbidity and mortality. It is an active process, sharing some similarities with atherosclerosis. Additional components of importance include calcification of the valve, LV hypertrophy and fibrosis.
Currently, there are no medical therapies that can avert disease progression.

Biological markers (such as cTnl) and advanced imaging modalities (such as cardiac magnetic resonance) that can demonstrate earliest evidence of LV decompensation may play a role in the timely detection of the transition from hypertrophy to heart failure, enabling prompt intervention.

The burden of AS is set to increase with an ageing population and the surgical risk is often prohibitive in such a cohort, especially if accompanied by multiple comorbidities. TAVI has already proven to be a comparable (if not superior) alternative to SAVR in high and intermediate-risk patients. Its effectiveness in low-risk patients will be addressed in imminent trials. 0

\section{References}

1 Vaslef SN, Roberts WC. Early descriptions of aortic valve stenosis. Am Heart J 1993; 125: 1465-74.

2 lung B, Baron G, Butchart EG et al. A prospective survey of patients with valvular heart disease in Europe: The Euro Heart Survey on Valvular Heart Disease. Eur Heart J 2003; 24: 1231-43.

3 Ross J, Braunwald E. Aortic stenosis. Circulation 1968; 38: 61-7.

4 Rosenhek R, Klaar U, Schemper M et al. Mild and moderate aortic stenosis. Natural history and risk stratification by echocardiography. Eur Heart J 2004; 25: 199-205.

5 Michelena HI, Desjardins VA, Avierinos JF et al. Natural history of asymptomatic patients with normally functioning or minimally dysfunctional bicuspid aortic valve in the community. Circulation 2008; 117: 2776-84.

6 Dweck MR, Boon NA, Newby DE. Calcific aortic stenosis: a disease of the valve and the myocardium. J Am Coll Cardiol 2012; 60: 1854-63.

7 Cowell SJ, Newby DE, Prescott RJ et al. Scottish Aortic Stenosis and Lipid Lowering Trial, Impact on Regression (SALTIRE) Investigators. A randomized trial of intensive lipid-lowering therapy in calcific aortic stenosis. N Engl J Med 2005; 352: 2389-97.

8 Cheng W, Li B, Kajstura J et al. Stretch-induced programmed myocyte cell death. J Clin Invest 1995; 96: 2247-59.

9 Leri A, Claudio PP, Li Q et al. Stretch-mediated release of angiotensin II induces myocyte apoptosis by activating p53 that enhances the local renin-angiotensin system and decreases the Bcl-2-to-Bax protein ratio in the cell. J Clin Invest 1998; 101: 1326-42.

10 Marcus ML, Koyanagi S, Harrison DG et al. Abnormalities in the coronary circulation that occur as a consequence of cardiac hypertrophy. Am J Med 1983; 75: 62-6.

11 Galiuto L, Lotrionte M, Crea F et al. Impaired coronary and myocardial flow in severe aortic stenosis is associated with increased apoptosis: a transthoracic Doppler and myocardial contrast echocardiography study. Heart 2006; 92: 208-12.

12 Hein S, Arnon E, Kostin S et al. Progression from compensated hypertrophy to failure in the pressure-overloaded human heart structural deterioration and compensatory mechanisms. Circulation 2003; 107: 984-91.

13 Taniguchi T, Morimoto T, Shiomii $\mathrm{H}$ et al. Initial surgical versus conservative strategies in patients with asymptomatic severe aortic stenosis. J Am Coll Cardiol 2015; 66: 2827-38.

14 Rosenhek R, Zilberszac R, Schemper M et al. Natural history of very severe aortic stenosis. Circulation 2010; 121: 151-6.
15 Chin CWL, Shah AS, Dweck MR et al. High-sensitivity troponin I concentrations are a marker of an advanced hypertrophic response and adverse outcomes in patients with aortic stenosis. Eur Heart J2014; 35: 2312-21.

16 Dweck MR, Joshi S, Murigu T et al. Midwall fibrosis is an independent predictor of mortality in patients with aortic stenosis. J Am Coll Cardiol 2011; 58: 1271-9.

17 Claeys MJ. High-sensitivity troponin: does it predict the shape of the iceberg underneath the surface? Eur Heart J 2014; 35: 22735.

18 Capoulade R, Magne J, Dulgheru R et al. Prognostic value of plasma B-type natriuretic peptide levels after exercise in patients with severe asymptomatic aortic stenosis. Heart 2014; 100: 1606-12.

19 d'Arcy J, Coffey S, Prendergast B et al. Large-scale community echocardiographic screening reveals a major burden of undiagnosed valvular heart disease in older people: the OxVALVE Population Cohort Study. Eur Heart J 2016; 37: 3515-22.

20 lung B, Baron G, Butchart EG et al. A prospective survey of patients with valvular heart disease in Europe: the Euro Heart Survey on valvular heart disease. Eur Heart J 2003; 24: 1231-43.

21 Society for Cardiothoracic Surgery in Great Britain and Ireland. Sixth National Adult Cardiac Surgical Database Report 2008. http://www. scts.org/_userfiles/resources/SixthNACSDreport2008withcovers. pdf

22 Bouma BJ, van Den Brink RB, van Der Meulen JH et al. To operate or not on elderly patients with aortic stenosis: the decision and its consequences. Heart 1999; 82: 143-8.

23 lung B, Cachier A, Baron G et al. Decision-making in elderly patients with severe aortic stenosis: why are so many denied surgery? Eur Heart J 2005; 26: $2714-20$.

24 Van Geldorp MW, Gameren M, Kappetein AP et al. Therapeutic decisions for patients with symptomatic severe aortic stenosis: room for improvement? Eur J Cardiothorac Surg 2009; 35: 953-7.

25 Charlson E, Legedza AT, Hamel MB. Decision-making and outcomes in severe symptomatic aortic stenosis. J Heart Valve Dis 2006; 15 : 312-21.

26 Cribier A, Eltchaninoff $\mathrm{H}$, Leon MB et al. Percutaneous transcatheter implantation of an aortic valve prosthesis for calcific aortic stenosis. First human case description. Circulation 2002; 106: 3006-8.

27 Leon MB, Smith CR, Mack M et al. PARTNER Trial Investigators. Transcatheter aortic-valve implantation for aortic stenosis in 
patients who cannot undergo surgery. N Engl J Med 2010; 363: 1597-607.

28 Smith CR, Leon MB, Mack MJ et al. PARTNER Trial Investigators Transcatheter versus surgical aortic-valve replacement in high-risk patients. N Engl J Med 2011; 364: 2187-98.

29 Adams DH, Popma JJ, Reardon MJ et al. U.S. CoreValve Clinical Investigators. Transcatheter aortic-valve replacement with a self expanding prosthesis. N Engl J Med 2014; 370: 1790-8.

30 Natarajan D, Makkar R, Prendergast B et al. Will this trial change my practice? Placement of Aortic Transcatheter Valves (PARTNER) II cohort A trial - transcatheter or surgical aortic-valve replacement in intermediate-risk patients. Eurolntervention 2016; 12: 805-8.

31 Leon MB, Smith CR, Webb JG et al. PARTNER 2 Investigators. Transcatheter or Surgical Aortic-Valve Replacement in IntermediateRisk Patients. N Engl J Med 2016; 374: 1609-20.

32 Thourani VH, Kodali S, Leon MB et al. Transcatheter aortic valve replacement versus surgical valve replacement in intermediate-risk patients: a propensity score analysis. Lancet 2016; 387: 2218-25.
33 Thyregod HG, Steinbrüchel DA, Søndergaard L. Transcatheter Versus Surgical Aortic Valve Replacement in Patients With Severe Aortic Valve Stenosis: 1-Year Results From the All Comers NOTION Randomized Clinical Trial. J Am Coll Cardiol 2015; 65: 2184-94.

34 Eggebrecht $\mathrm{H}$, Mehta RH. Transcatheter aortic valve implantation (TAVI) in Germany 2008 - 2014: on its way to standard therapy for aortic valve stenosis in the elderly? Eurolntervention 2016; 11: 1029-33.

35 Oguri A, Yamamoto M, Mouillet $G$ et al. FRANCE 2 Registry Investigators. Clinical outcomes and safety of transfemoral aortic valve implantation under general versus local anesthesia: subanalysis of the French Aortic National CoreValve and Edwards 2 registry. Circ Cardiovasc Interv 2014; 7: 602-10. 